\title{
On Two Integrable Cellular Automata $\star \star$
}

\author{
Alexander Bobenko ${ }^{1, \star}$, Martin Bordemann ${ }^{2}$, Charlie Gunn ${ }^{1}$, Ulrich Pinkall ${ }^{1}$ \\ 1 Fachbereich Mathematik, Technische Universität Berlin, Strasse des 17. Juni 136, D-10623 Berlin, \\ Germany \\ 2 Fachbereich Physik der Universität Freiburg, Hermann-Herder-Str. 3, D-79104 Freiburg, Germany
}

Received: 16 July 1992/in revised form: 26 February 1993

\begin{abstract}
We describe two simple cellular automata (CA) models which exhibit the essential attributes of soliton systems. The first one is an invertible, 2-state, 1dimensional $\mathrm{CA}$ or, in other words, a nonlinear $\mathbf{Z}_{2}$-valued dynamical system with discrete space and time. Against a vacuum state of 0 , the system exhibits light cone particles in both spatial directions, which interact in a soliton-like fashion. A complete solution of this system is obtained. We also consider another CA, which is described by the Hirota equation over a finite field, and present a Lax representation for it.
\end{abstract}

\section{Introduction}

Cellular automata (CA) have become increasingly popular models for physical systems [9]. CA are regular grids of finite state automata each of whose states at successive time steps is determined uniformly by the states of some finite neighborhood. In the simplest case the grid is a one-dimensional array. This dimension can be referred to as the $x$-dimension and CA can be described as a dynamical system in discret space and time whose field variables take only finitely many values. CA were found with coherent particle-like structures. Some of these particles scatter as solitons [7, 1].

We suggest new time-reversable CA, which we call soliton systems, because they possess the usual features of the integrable systems. Let us consider a diagonally oriented lattice and some horizontal stairway $S$ on it (shaded in Fig. 1).

We consider a field $v$ taking values in some finite set. $\mathscr{F}$ at each face of $S$ and which obey an evolution equation of the form

$$
v_{d}=F\left(v_{l}, v_{u}, v_{r}\right)
$$

(We direct time down)

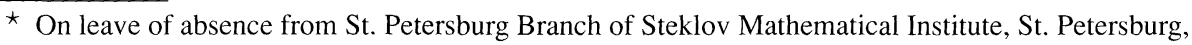
Russia

$\star \star$ Supported by Deutsche Forschungsgemeinschaft, Sonderforschungsbereich 288
} 


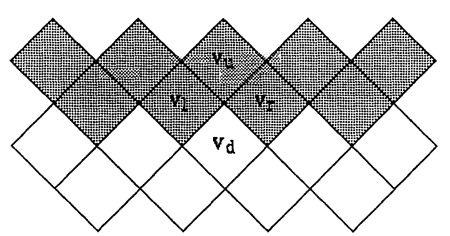

Fig. 1. Diagonally oriented lattice with evolution at one vertex

\section{The Simplest Soliton System $\left(\mathrm{Z}_{2}\right.$-System $)$}

\subsection{Description of the System}

Let us consider the simplest case $\mathscr{F}=\mathbf{Z}_{2}=\{0,1\}$, when $v$ 's at faces are equal to 0 or 1 . It is clear that there exist 256 possible choices for $F$, since each $F$ is a rule for assigning a 0 or 1 to each of the possible $2^{3}=8$ inputs. We name these inputs by reading the sequence of vertex values $\left(v_{l}, v_{u}, v_{r}\right)$ as a binary number, with $v_{l}$ the least significant bit. Figure 2 shows these 8 possible combinations $c_{i}$, $i \in\{0,1,2,3,4,5,6,7\}$.

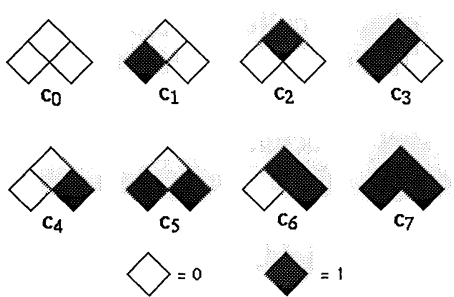

Fig. 2. The eight possible $\left(v_{l}, v_{u}, v_{r}\right)$ configurations

We reduce this set of possibilities by enforcing two symmetry constraints:

Left-right symmetry: $F\left(v_{l}, v_{u}, v_{r}\right)=F\left(v_{r}, v_{u}, v_{l}\right)$.

Up-down symmetry: If $v_{d}=F\left(v_{l}, v_{u}, v_{r}\right)$, then $F\left(v_{l}, v_{d}, v_{r}\right)=v_{u}$.

These constraints reduce our search to 8 possibilities, since they force:

$$
\begin{gathered}
c_{4}=c_{1}, \quad c_{6}=c_{3}, \\
F\left(c_{2}\right) \neq F\left(c_{0}\right), \quad F\left(c_{3}\right) \neq F\left(c_{1}\right), \quad F\left(c_{7}\right) \neq F\left(c_{5}\right) .
\end{gathered}
$$

Expressed as 8-bit numbers, where the value of the $i^{\text {th }}$ bit is $F\left(c_{i}\right)$, these eight rules are 204 (11001100), 51 (00110011), 150 (10010110), 105 (01101001), 108 (01101100), 147 (10010011), 54 (00110110), 201 (11001001). Warning: These binary numbers have to be read from the right to the left in order to get the values of $c_{0}, c_{1}, \ldots, c_{7}$.

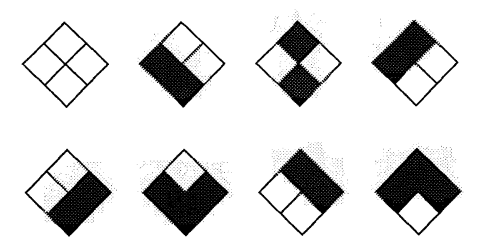

Fig. 3. Rule 54 
However, there is an alternative form for these maps which we demonstrate now. Consider the maps $v_{d}=v_{u}+f\left(v_{l}, v_{r}\right)$, where $f\left(v_{l}, v_{r}\right)=f\left(v_{r}, v_{l}\right)$, i.e., $f$ has left-right symmetry. Such an $f$ is determined by its values on the pairs $((0,0),(0,1),(1,1))$. There are eight such maps, and defining $F_{i}=f_{i}+v_{u}$ we recover the above rules:

$$
\begin{aligned}
\text { 204: } & f_{0}=0, & 108: & f_{4}=v_{l} v_{r}, \\
\text { 51: } & f_{1}=1, & 147: & f_{5}=v_{l} v_{r}+1, \\
150: & f_{2}=v_{l}+v_{r}, & 54: & f_{6}=v_{l} v_{r}+v_{l}+v_{r}, \\
105: & f_{3}=v_{l}+v_{r}+1, & 201: & f_{7}=v_{l} v_{r}+v_{l}+v_{r}+1,
\end{aligned}
$$

Hence we have found polynomial expressions for all the maps which satisfy the desired symmetry constraints.

Notice that the $f_{0}, f_{1}, f_{2}$, and $f_{3}$ are affine, hence the dynamics are boring. Of the remaining four maps, we can identify them in pairs by swapping the roles of 0 and 1 in each rule. Then $F_{4} \simeq F_{7}$ and $F_{5} \simeq F_{6}$. We turn our attention to $F_{6}$,

$$
v_{d}+v_{u}=v_{l} v_{r}+v_{l}+v_{r}(\bmod 2)
$$

which we choose in preference to $F_{5}$ since it has a 0 vacuum state, i.e., a state of all 0 's is preserved under the system. ${ }^{1}$

The system equivalent to $F_{4}$ was considered in [1]. It does not possess particle-like structures against the vacuum: computation shows that the particle world-lines (i.e. sequence of 1's or black squares) are approximately vertical, i.e. at rest.

From now on in all figures faces with $v=1$ are shaded or colored black. Vacuum faces $(v=0)$ will always be white. Figures 4 and 5 show typical evolutions of the $F_{6}$ rule. Figure 4 has a region of random initial conditions within a background of 0 's; Fig. 5 shows the collision of two oppositely directed particles. Notice that the two particles pass through one another completely, but with a time delay (phase-shift) characteristic of solitons.

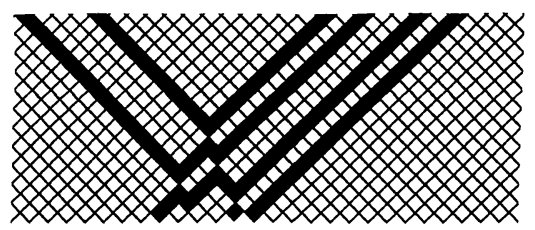

Fig. 4. Evolution of rule 54 from random initial configuration

Fig. 5. Collision of two particles

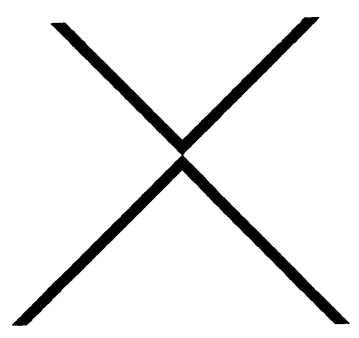

Since 0 is so closely tied in the popular imagination to the concept of vacuum 


\subsection{Conservation Laws}

We assign a set of arrows to each stairway. A 3-block is a set of three successive faces in a larger string. In the case of the stairway, there are 16 distinct 3-blocks: 8 are given by the $c_{\imath}$ introduced above; the other 8 are given by the blocks, which can be gotten from the $c_{\imath}$ by inverting, so that they point down instead of up. We assign arrows to a stairway based on the occurrence of certain 3-blocks as shown in Fig. 6. The other 11 3-blocks not shown have no arrows associated with them.

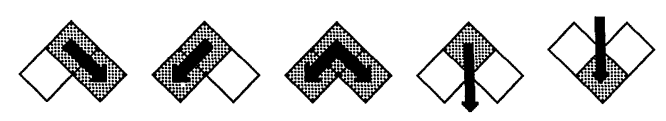

Fig. 6. Arrows are assigned for these 3-blocks in the stairway

The arrows always connect black points. Intuitively, the vertical arrow represents a combination of 2-particles, one left-going and one right-going.

Theorem 1. The only possible configurations for a vertex in the arrow graph are shown in Fig. 7.

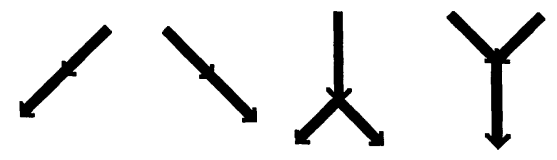

Fig. 7. Possible arrow configurations

This follows directly from the above $F_{6}$ rule (cf. Fig. 3).

Corollary 1. Arrows are preserved in successive stairways.

Here, we mean that the vertical arrow should be thought of as the union of one left-going and one right-going particle. To establish the theorem it suffices to examine all the possibilities.

\subsection{Cauchy Problem, Asymptotic Behavior, and Scattering of Solitons}

Theorem 1 shows that arrows (solitons) interact only pairwise and only in a very simple way (Fig. 5). This fact gives the solutions of the Cauchy problem.

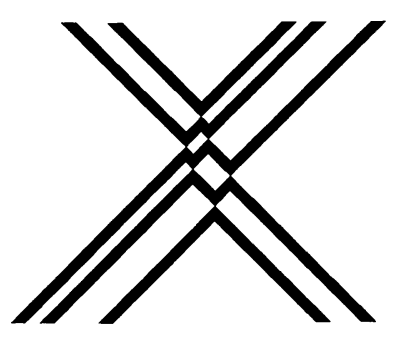

Fig. 8. Interaction of two soliton beams 
In particular for a localized Cauchy (finite number of $1 s$ ) we get the following asymptotic behaviour.

Corollary 2. A right-(left-)going particle A will be displaced (delayed) by the number of left-(right-)going particles to its right (left).

Let the number of left-going particles to the right of $\mathrm{A}$ be denoted by $\mathrm{B}$. Then each member of B will intersect $\mathrm{A}$ and such an intersection, declares the arrow theorem, will delay both particles for one time step in its directed motion (see Fig. 5).

Figure 8 shows the scattering of two soliton beams. Again the pairwise character of interaction proves that if $n$ solitons go left and $m$ solitons go right, then after the collision these two beams as a whole get phase-shifts $m$ and $n$ respectively. The intervals between the solitons of the beam are preserved.

\section{The Hirota Equation over Finite Fields and its Lax Representation}

Now we describe a dynamical system over a finite field, which obeys the Lax representation. The Hirota equation [5]

$$
v_{u} v_{d} v_{l} v_{r}+k\left(v_{u} v_{d}-v_{l} v_{r}\right)=1
$$

can be represented in the Lax form [8]

$$
M\left(\lambda, v_{r}, v_{d}\right) L\left(\lambda, v_{d}, v_{l}\right)=L\left(\lambda, v_{r}, v_{u}\right) M\left(\lambda, v_{u}, v_{l}\right)
$$

with the matrices

$$
\begin{gathered}
L\left(\lambda, v_{1}, v_{2}\right)=\left(\begin{array}{cc}
v_{1} / v_{2} & \lambda \\
\lambda & v_{2} / v_{1}
\end{array}\right), \\
M\left(\lambda, v_{1}, v_{2}\right)=\left(\begin{array}{cc}
k & \lambda^{-1} v_{1} v_{2} \\
\lambda^{-1}\left(v_{1} v_{2}\right)^{-1} & k
\end{array}\right) .
\end{gathered}
$$

The existence of the Lax representation allows us to apply the powerful machinery of the soliton theory and as a result, for example, to find integrals of motion and to solve the periodic problem [2].

We rewrite the Hirota equation in the following form:

$$
v_{u} v_{d}=\frac{1+k v_{l} v_{r}}{k+v_{l} v_{r}}
$$

Previous investigations $[2,8]$ have been done for the case where the $v$ 's are complex numbers, but the above calculations make sense in the following more general context:

i) The $v$ 's take values in a subgroup $S^{1}$ of the multiplicative group $\mathbf{F}_{*}$ of some field F,

ii) $k \in \mathbf{F}$ is such that the Moebius transformation

$$
v \rightarrow \mathscr{M}(v)=\frac{1+k v}{k+v}
$$

maps $S^{1}$ into itself. 
The easiest method to meet these requirements for a finite field $\mathbf{F}$ is the following. Start with the prime field $\mathbf{Z}_{\mathbf{p}}$ and a non-square $b \in \mathbf{Z}_{\mathbf{p}} \cdot{ }^{2}$ Then define

$$
\mathbf{F}=\left\{x+\sqrt{b} y \mid x, y \in \mathbf{Z}_{\mathbf{p}}\right\} .
$$

$\mathbf{F}$ is a quadratic extension of $\mathbf{Z}_{\mathbf{p}}$ and has $p^{2}$ elements. For $v=x+\sqrt{b} y$ we define $\bar{v}=x-\sqrt{b} y \cdot v \rightarrow \bar{v}$ is an involutive field automorphism whose fixed point set is $\mathbf{Z}_{\mathbf{p}} \subset \mathbf{F}$. Define

$$
S^{1}=\{v \in \mathbf{F} \mid v \bar{v}=1\} .
$$

Obviously $S^{1}$ is a multiplicative subgroup of $\mathbf{F}$ satisfying $S^{1} \cap \mathbf{Z}_{\mathbf{p}}=\{-1,1\}$ if $p \geq 3$.

Lemma 1. For any odd prime $p$ we have $S^{1}=\sigma\left(\mathbf{Z}_{\mathbf{p}} \cup\{\infty\}\right)$ where $\sigma: \mathbf{F} \cup\{\infty\} \rightarrow$ $\mathbf{F} \cup\{\infty\}$ is the Moebius transformation

$$
z \mapsto \sigma(z)=\frac{\sqrt{b}+z}{\sqrt{b}-z},
$$

$\sigma(\sqrt{b})=\infty$, and $\sigma(\infty)=-1$.

Thus $S^{1}$ has $p+1$ elements.

Proof. $\sigma$ is quickly computed to be injective since $p \neq 2$. Because everything is finite it is also bijective. Now $\sigma(\infty) \overline{\sigma(\infty)}=1$ by definition, and

$$
\sigma(z) \overline{\sigma(z)}=\frac{\sqrt{b}+z-\sqrt{b}+z}{\sqrt{b}-z-\sqrt{b}-z}=1
$$

which proves the lemma. Q.E.D.

To meet the above requirement ii) it is then sufficient that $k \in \mathbf{Z}_{\mathbf{p}}$ ( $k$ is "real") and $k \notin\{-1,1\}$. For $p=2,3$ no such $k$ exists, so the simplest example is given by $p=5, k=b=2$ (the cases $k=-2$ or $b=-2$ are not essentially different). In this case $S^{1}$ is given by

$$
\left\{1, q, q^{2}, q^{3}, q^{4}, q^{5}\right\}=\{1,-2+2 \sqrt{2}, 2+2 \sqrt{2},-1,2-2 \sqrt{2},-2-2 \sqrt{2}\} .
$$

The correspondence $j \rightarrow q^{j}$ yields an isomorphism of $S^{1}$ (as an abelian group) with the cyclic group $\mathbf{Z}_{6}$. This is no coincidence since the multiplicative group of a finite field (and thus any subgroup thereof) is known to be cyclic (cf. e.g. [6], p. 132, Theorem 2.18). The way how the Moebius transformation (3) acts on $S^{1}$ is indicated in Fig. 9 (the points \pm 1 are fixed).

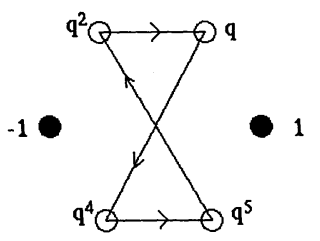

Fig. 9. The Moebius transformation $\mathscr{H}(v)$ on $S^{1}$

$\sqrt{2 \text { For instance, }}-1$ is known to be a non-square in $\mathbf{Z}_{\mathbf{p}}$ iff $p=3 \bmod 4$ 
Theorem 2. The equation

$$
v_{u} v_{d}=\mathscr{M b}\left(v_{l} v_{r}\right)
$$

describes a $\mathrm{CA}$ on the set $\mathscr{F}=S^{1}$, possessing the Lax representation (1).

As usual, $L$ and $M$ matrices are associated with the corresponding edges. For the periodical problem a transfer matrix $T(\lambda)$ is defined as a product of $L$ 's and $M$ 's along the consecutive edges of the stairway.

$$
T=L_{N} M_{N} \ldots L_{1} M_{1} \text {. }
$$

Corollary 3. $\operatorname{tr} T(\lambda)$ is preserved under the evolution and generates integrals of motion of the system.

Computer experiments of the above system exhibit a much more complicated behavior than in the $\mathbf{Z}_{2}$ case.

Remark. Equation (2) is invariant with respect to two (global) gauge transformations $\left(a, b \in S^{1}\right)$ :

i) $v_{l} \mapsto a v_{l}, v_{r} \mapsto a^{-1} v_{r}$,

ii) $v_{u} \mapsto b v_{u}, v_{d} \mapsto b^{-1} v_{d}$.

i.e. i) acts on a fixed sublattice with double lattice spacing and ii) acts on its (vertex) complement, the distribution of $a, a^{-1}$ and $b, b^{-1}$ being chequed on each sublattice, respectively. There is a gauge invariant version of the Hirota equation, having a natural geometrical interpretation ([2]) which is called the discrete sine-Gordon equation. In order to derive it we consider a bigger fragment of the lattice (see Fig. 10) and introduce fields $u \in S^{1}$ on the dual lattice, which are products of two $v$ 's at two horizontally neighbouring vertices

$$
u_{l}=v_{3} v_{4}, u_{r}=v_{4} v_{5}, u_{d}=v_{6} v_{7}, u_{u}=v_{1} v_{2} .
$$

The Hirota equation for the $v$ 's implies

$$
v_{1} v_{6}=\mathscr{H}\left(u_{l}\right), v_{2} v_{7}=\mathscr{M}\left(u_{r}\right) .
$$

Multiplying these two equations we get the discrete sine-Gordon equation

$$
u_{u} u_{d}=\mathscr{M}\left(u_{l}\right) \mathscr{M}\left(u_{r}\right)
$$

which describes a CA over $S^{1}$.

Remark. We should like to mention two more papers dealing with integrable CA: In the article [4] a good survey of results on CA with particle-like behaviour is presented.

Fig. 10. Bigger fragment of the lattice

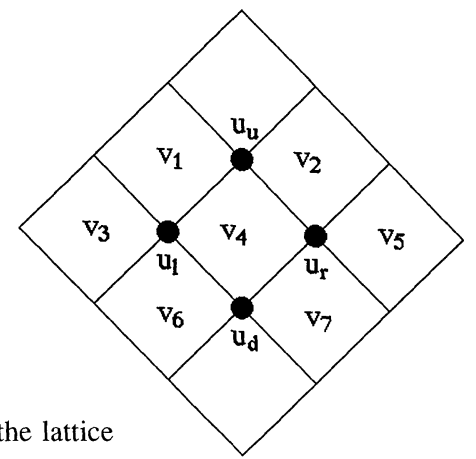


Moreover, an example of a CA admitting a Lax representation was recently obtained in [3]. It is based on a discrete version of the Schrödinger equation.

Acknowledgements. The authors would like to thank Ulrich Brehm and Detlef Hardorp for helpful discussions.

\section{References}

1. Ablowitz, M., Keizer, J., Takhtajan, L.: Stable multi-state time-reversible cellular automata with rich particle content. Preprint University of Colorado (1991)

2. Bobenko, A., Pinkall, U.: Discrete surfaces with constant negative curvature and the Hirota equation (in preparation)

3. Bruschi, M., Santini, P.M., Ragnisco, O.: Integrable cellular automata. Phys. Lett. A 169, 151 (1992)

4. Fokas, A.S., Papadopoulou, E.P., Saridakis, Y.G.: Soliton cellular automata. Physica D 41, $297-$ 321 (1990)

5. Hirota, R.: Nonlinear partial difference equations. III. Discre Sine-Gordon equation. J. Phys. Soc. Japan 43:6, 2079-2086 (1977)

6. Jacobson, N.: Basic algebra. I. San Francisco, 1985

7. Park, J., Steglitz, K., Thurston, W.: Soliton-like behaviour in automata. Physica 19D, 423 (1986)

8. Quispel, G., Capel, H., Papageorgiou, V., Nijhoff, F.: Integrable mappings derived from soliton equations. Preprint LaTrobe University (1990)

9. Wolfram, S.: Theory and applications of cellular automata. Singapore: World Scientific 1986

Communicated by N. Yu. Reshetikhin 